\title{
M-mode echocardiography in the developing human
}

\section{fetus}

\author{
LINDSEY D ALLAN, MICHAEL C JOSEPH, EDWARD G C A BOYD, \\ STEWART CAMPBELL, MICHAEL TYNAN
}

From Guy's Hospital, and King's College Hospital, London

SUMMARY The M-mode echocardiogram can be obtained in the developing human fetus from around the 16th week of gestation until term. Errors in interpretation of the M-mode echocardiogram can be avoided by concurrent two dimensional fetal cardiac examination. The pattern of motion of the mitral, tricuspid, aortic, and pulmonary valves and ventricular wall motion can be studied. Motion of the foramen ovale flap, which is characteristic in fetal life, can also be observed. Using the $M$-mode tracing, measurement data for six variables were made and growth charts constructed. The variables recorded were septal and left ventricular wall thickness, right and left ventricular internal dimension in diastole, aortic root dimension, and left atrial internal dimension. The measurements were made in normal pregnancies where the fetal heart was structurally normal. Confidence limits for each measurement were derived for gestational ages between 16 and 39 weeks.

The anatomy of the normal fetal heart can be visualised using realtime two dimensional echocardiography from around the 16 th week of gestation until term. ${ }^{12}$ During our continuing echocardiographic study for the prenatal detection of cardiac abnormalities it became apparent that it was also possible to record an $M$-mode echocardiogram. The M-mode allows the study of the pattern of motion of cardiac valves and wall movement. It allows clear definition of endocardial surfaces. These are difficult to define on a realtime frozen frame image which has been used by other authors for the acquisition of measurement data. ${ }^{1}$ Measurements can be more precisely timed within the cardiac cycle on the M-mode echocardiogram. Standard measurements can be made that can be compared with postnatal data. In addition, functional variables can be derived as from the postnatal echocardiogram.

Once the anatomical structures and the orientation of the fetal heart have been identified it is possible to select the $M$-mode recording to be made by locating the M-line (ATL Mark III) through the structures to be studied. It is essential that two dimensional identification precedes the $M$-mode examination. This is because it can be impossible to differentiate the aortic from the pulmonary valve or the mitral

This research has been supported by the British Heart Foundation.

Accepted for publication 2 March 1982 from the tricuspid valves by their M-mode motion alone. It can also be difficult to differentiate right from left ventricular wall motion, especially in the early fetal heart.

Detailed fetal cardiac measurements are not as yet available and therefore we have compiled data for pregnancies between 16 weeks gestation and term. The measurements made were septal and left ventricular wall thickness, right and left ventricular internal dimensions, aortic root and left atrial dimensions.

\section{Subjects and methods}

Fetal M-mode echocardiograms have been obtained in 254 subjects. Ethical committee approval for this study was obtained. Originally the aortic root dimension only was measured in 54 subjects. It was subsequently realised that a complete $M$-mode echocardiogram could be attempted. This was done in 200 subjects. An Advanced Technical Laboratories Mark III sector scanner with a $3 \mathrm{MHz}$ transducer was used. The two dimensional pictures were recorded on a Sanyo VTC 7100 video cassette recorder and the M-mode on a Honeywell black and white stripchart recorder operated at $50 \mathrm{~mm} / \mathrm{s}$.

In 48 cases a fetal electrocardiogram was also recorded on the $\mathrm{M}$-mode tracing, using a purpose built isolated fetal electrocardiographic preamplifier 
connected to three abdominal electrodes.

The M-mode echocardiogram was studied and measurements made only in normal pregnancies where no structural abnormality of the fetal heart was apparent. The fetal age was determined by biparietal diameter measurements made early in pregnancy, that is before 20 weeks gestation, when this measurement is the most reliable determinant of fetal age. ${ }^{3}$ Estimated gestational age by this means was felt to be the most useful correlate with cardiac measurements as the majority of our data lay between 16 and 28 weeks gestation. In this age range other fetal measurements such as derived fetal weight are less accurate.

Two dimensional echocardiographic identification of fetal cardiac chambers was performed initially. This is essential since it cannot be assumed that structures visualised closest to the transducer are anterior in the fetus. Nor can it be assumed that the fetus has a normal heart. Abnormalities of connections and relations must be excluded. Fortunately the unobstructed access to the fetal heart allows this to be accomplished using morphological criteria. The right ventricle is differentiated from the left by the coarser right ventricular trabeculations and the more apical insertion of the septal leaflet of the tricuspid valve. Fig. 1 shows a still frame demonstrating these features, the angulation of the tricuspid valve indicating its more apical septal insertion. This differentiating feature is seen more easily in the moving two dimensional image. The aorta is identified by showing its continuity from the left ventricle to the ascending aorta, aortic arch, and its branches. The pulmonary artery must be shown to connect the right ventricle to the ductus arteriosus. Thus, the aortic valve is differentiated from the pulmonary valve by identification of the arteries in which they reside. The entry of the systemic and pulmonary venous channels to the appropriate atria can also be seen. After the basic structure of the heart was established and cardiac normality identified the $M$-line was then directed through the valve or chamber to be studied.

The pulmonary and aortic valves and the foramen is ovale flap were readily visualised directly and the $\overrightarrow{0}$ M-line positioned through them. A suitable recording for measuring the ventricular chambers can only be obtained in the four chamber view if the $M$-line can be located perpendicular to the ventricular septum. This is only possible if the fetus lies with either shoulder towards the transducer. If the fetal position is unsuitable a short axis view of both ventricles should be obtained as seen in Fig. 2. It is then possible to sweep from the atrioventricular valves down into the body of both ventricles to obtain a measurement of the minor axis of both ventricles.

The measurements made included the following.

\section{AORTIC ROOT}

The maximum dimension of the aortic root was measured only in an orientation when the aortic valve closure line could be seen as in Fig. 3.

\section{LEFT ATRIAL DIMENSION}

The maximum dimension of the left atrium was measured. Since the apparent size of the left atrium is dependent on the orientation of the fetus, left atrial $\vec{\partial}$ measurements were only made when there was no

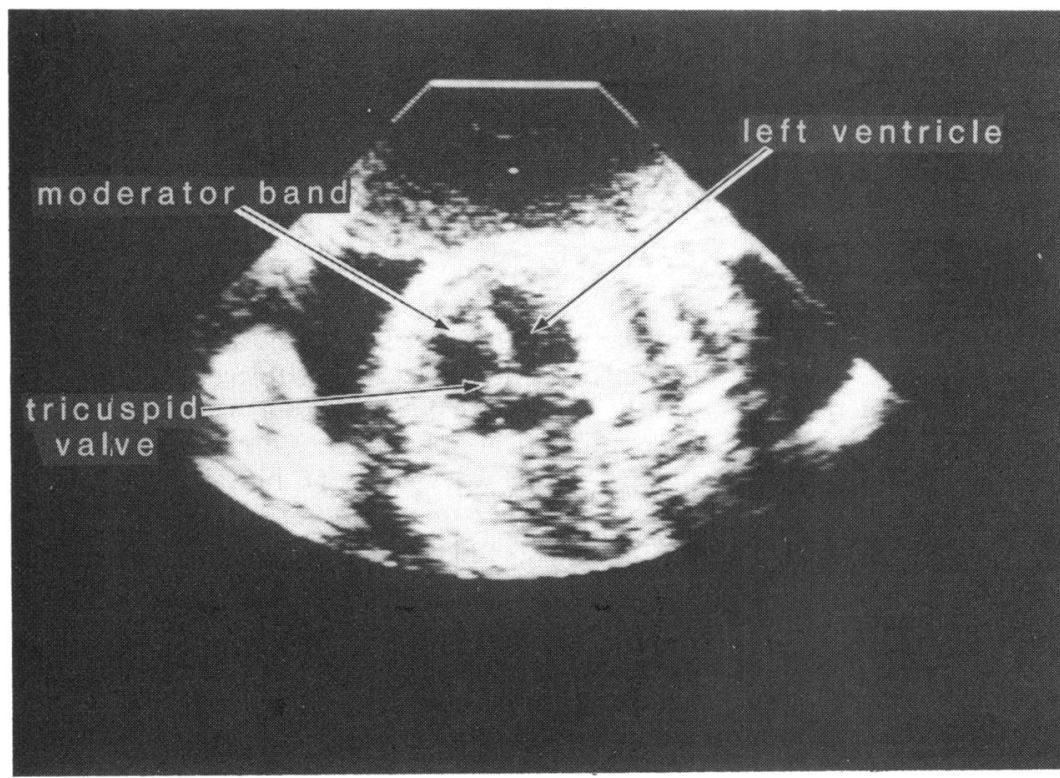

Fig. 1 A four chamber projection of a normal fetal heart showing increased echoes in the apex of the right ventricle and a more apically $N$ inserted atrioventricular valve in this chamber. 


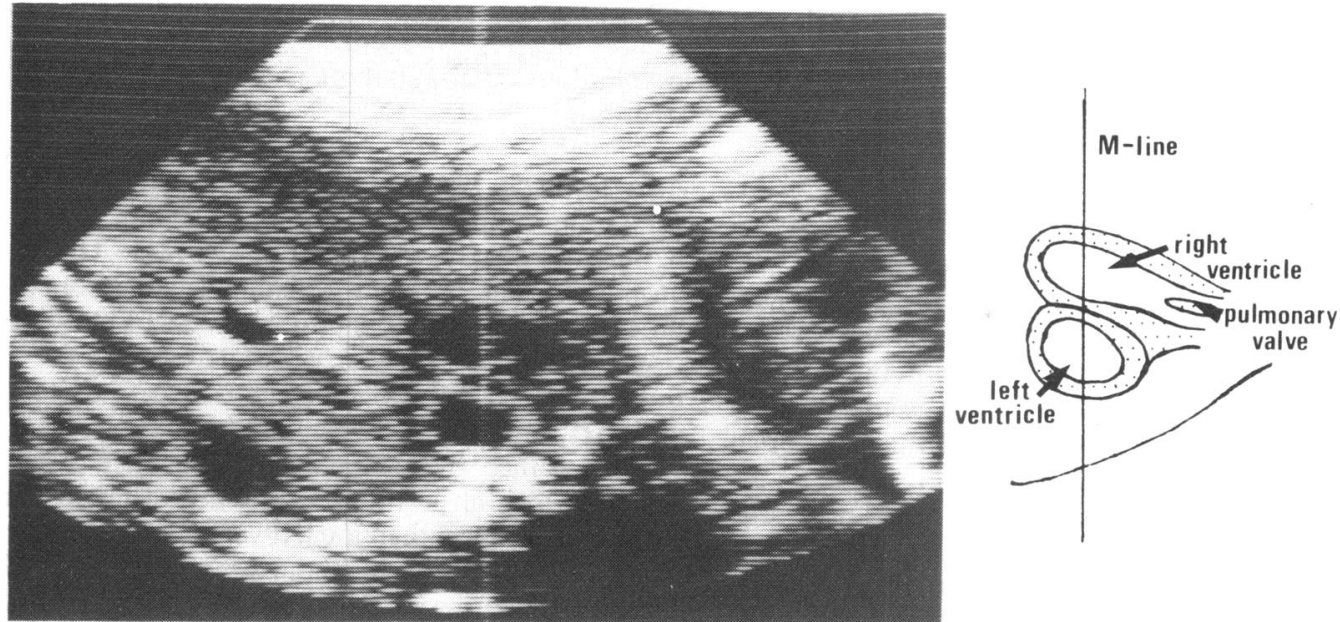

Fig. 2 The M-mode line is located through the two ventricular chambers seen on a short axis projection. The fetal head lies to the right of the picture, the diaphragm and fetal liver to the left of the fetal heart.

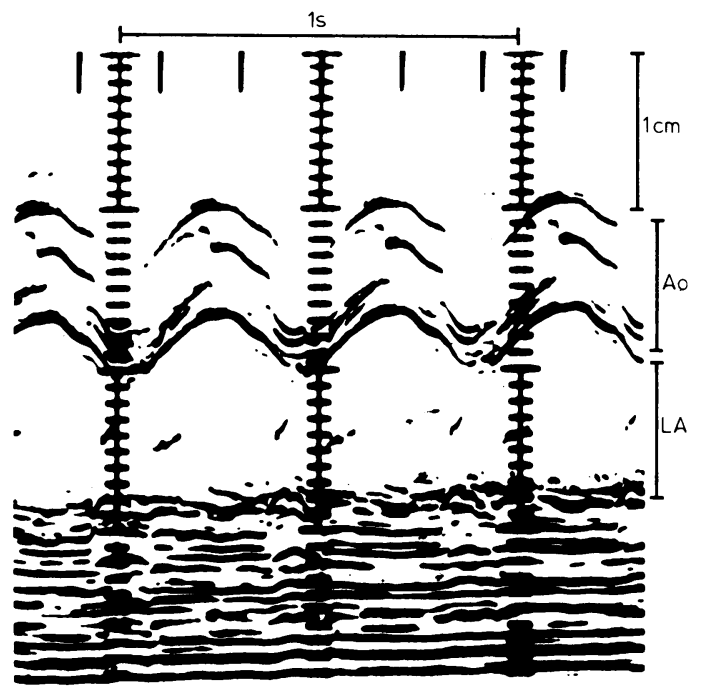

Fig. 3 The aortic root, aortic valve, and left atrium of a 29 week fetus. The structure moving within the left atrium is the foramen ovale flap.

obliquity of the M-line to the left atrium. This limited measurements to those subjects in whom a true short axis or long axis view was obtained.

\section{RIGHT AND LEFT VENTRICULAR DIMENSIONS}

End-diastolic measurements were made at the maximum ventricular dimension. This is recognised to be a slightly larger dimension than that obtained by timing the measurement to coincide with the $R$ wave of the electrocardiogram. This dimension was chosen because the fetal electrocardiogram was only recorded in 48 of the 200 subjects in whom these measurements were made. Measurements were made between the endocardial surfaces just apical to the recording of the mitral or tricuspid valves. To measure both right and left ventricular dimensions the short axis view was used. In some instances this could not be obtained and the long axis view of the left ventricle was used. This latter view is valid for left ventricular measurements but since the beam crosses only the outlet portion of the right ventricle, right ventricular dimensions were not estimated when only the long axis view was available.

\section{SEPTAL AND POSTERIOR LEFT VENTRICULAR WALL THICKNESS}

These measurements were taken from recordings made for ventricular dimension measurement and were made at the same point as that taken for enddiastolic dimension. Fig. 4 shows a suitable recording for measurement of right and left ventricular internal dimensions, and septal and posterior left ventricular wall thickness.

All the measurements were made to the nearest $0.5 \mathrm{~mm}$. No ventricular measurement was made when there was doubt concerning the definition of endocardial surfaces. The results were analysed statistically by regression analysis and $95 \%$ confidence limits were derived.

Left and right ventricular ejection times were measured by direct observation of the aortic and pulmonary valves when both opening and closure were adequately visualised. 


\section{Results}

These are divided into the descriptive echocardiographic data and the measurement data.

\section{DESCRIPTIVE DATA}

\section{Arterial valve and foramen ovale flap motion}

There are several echocardiographic sections in which the pulmonary or aortic valve may be visualised. ${ }^{2}$ Whichever orientation is used to perform the M-mode record the arterial valves appear the same; with a "box" shape in systole and sloping closure line often with what appears to be an "A" dip. Fig. 5 shows the pulmonary valve motion in a 19 week fetus. Below the $M$-mode trace the combined maternal and fetal electrocardiogram is recorded from midline external abdominal electrodes. Fig. 3 shows the familiar aortic valve "box" appearance in a 29 week fetus with the left atrium lying posteriorly. The echoes within the left atrium were shown on the two dimensional scan to originate from the foramen ovale flap. This has a characteristic biphasic movement throughout each cardiac cycle, seen more clearly in Fig. 6. This pattern of motion was observed in every fetus studied. Closure, or approximation of the foramen ovale flap to the atrial septum, is related to atrial systole. The foramen ovale then opens and closes more gently dur-

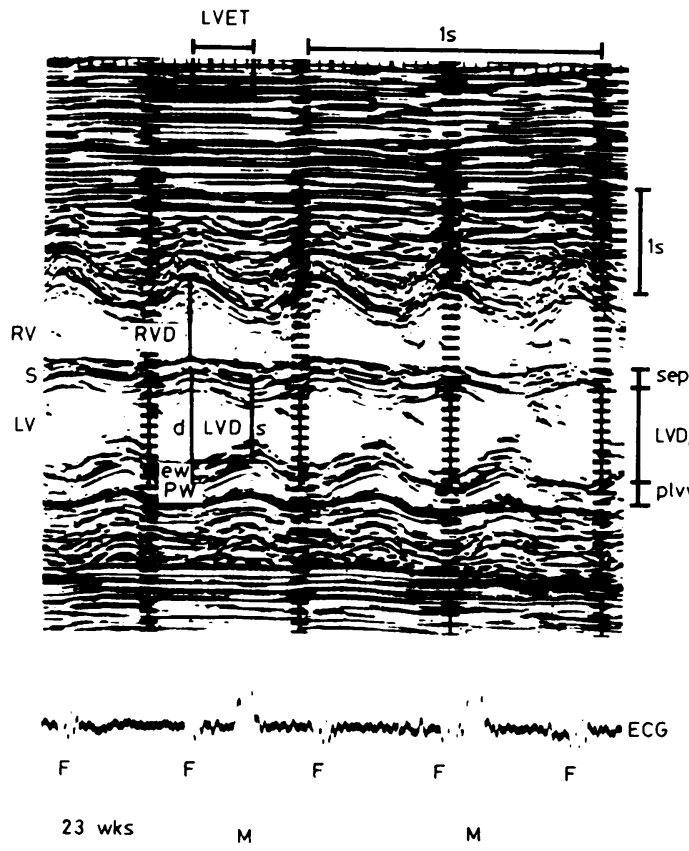

Fig. 4 A suitable $M$-mode echocardiogram for measuring right and left ventricular chamber sizes and septal and left ventricular wall thickness. ing ventricular systole. Opening again occurs and the cycle is then repeated with the sharp closure of atrial systole. Left atrial wall motion can also be seen in this recording.

\section{Atrioventricular valve and ventricular wall motion}

Fig. 7 shows the $M$-mode echocardiogram sweeping between both atrioventricular valves. The two atrioventricular valves are indistinguishable from each other by their M-mode appearance alone. Differentiation of the atrioventricular valves depends on the two dimensional identification of ventricular morphology as described in the Methods section. Some indication may be gained by looking at right ventricular wall motion which always appears to have a much more undulating pattern than the left ventricular wall in the normal fetus (Fig. 6). In all the fetuses the ventricular septum moved towards the posterior left ventricular wall in systole. In addition, by sweeping up from the atrioventricular valves to the aorta, mitral-aortic continuity and tricuspid-aortic discontinuity can be shown. A mitral-aortic sweep is seen in Fig. 8.

\section{MEASUREMENT DATA}

The aortic root could be clearly identified and a measurement was obtained in all 200 subjects. In 54 earlier subjects a measurement of the aortic root was made when the feasibility of adequate $M$-mode recordings was being assessed and a recording of the aortic root alone was attempted. The additional measurements were included in the statistical data. In 45 subjects both arterial valves were visualised adequately for estimates of both right and left ventricular ejection times to be measured. Estimates for both were similar, being $200 \mathrm{~ms}$ with a range of $\pm 40 \mathrm{~ms}$. Since left atrial position depends entirely on the fetal cardiac orientation it was only reliably recorded in 107 of the subjects. The incidence of success in achieving an adequate left atrial measurement did improve, however, as the study progressed.

Adequate recordings for both left and right ventricular dimensions were achieved in 167 of the 200 subjects, with fewer failures in the latter part of the study. In a further eight subjects the septal thickness, left ventricular internal dimension, and posterior left ventricular wall thickness could be measured in a long axis view of the left ventricle, but no right ventricular measurement could be made. In a further three subjects the septal endocardial surfaces were visualised but not the posterior left ventricular wall; thus, in a total of 178 subjects septal thickness was measured.

Regression analysis showed that the best correlation for each measurement to gestational age was a straight line of the form $y=m x+b$. With some measurements there was an indication of a deviation from linearity in the later weeks of pregnancy but this was small, 

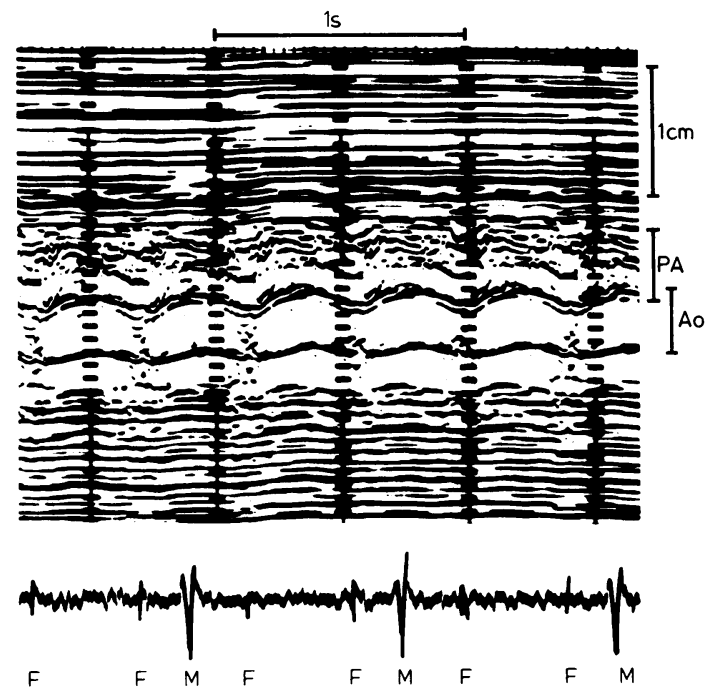

Fig. $5 M$-mode echocardiogram of the pulmonary valve motion in a 19 week fetus. The fetal electrocardiogram is also shown. $F$ etal complexes are marked $F$, maternal $M$.
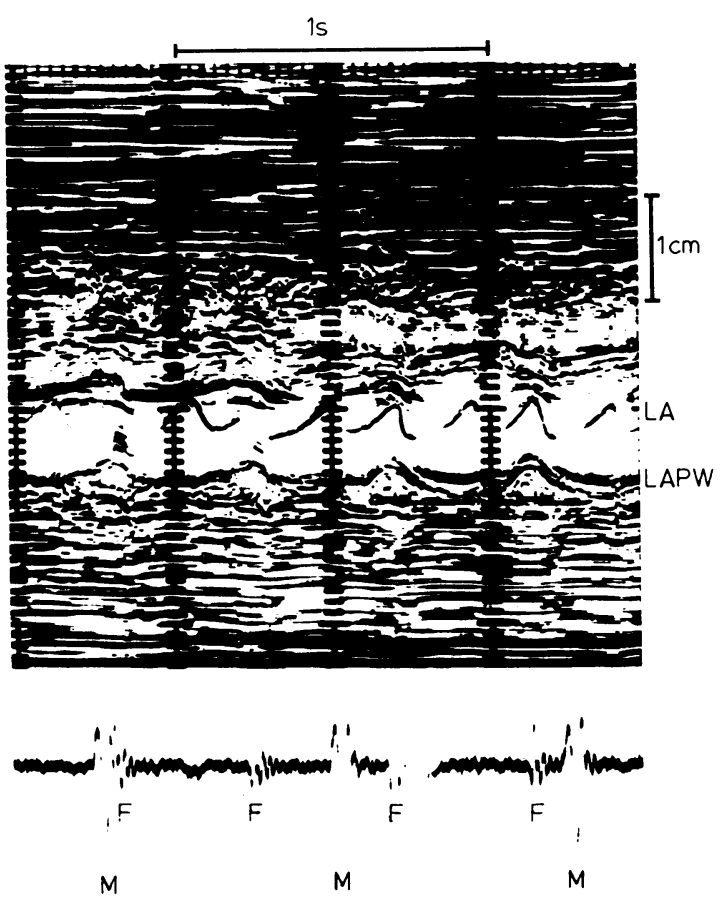

Fig. 6 Left atrial wall motion, and foramen ovale flap motion in a 22 week fetus. The foramen ovale flap moves away from the atrial septum twice within each cardiac cycle. inconsistent, and based on few data points. The "flared" $95 \%$ confidence limits for individual measurements (Table) were only slightly wider at the extremes of the range than at the mean gestation; this again argues against a significant deviation from linearity. Straight $95 \%$ confidence limits defined by twice the standard error of the estimate of y (SEE $y \times 2$ ) were in close agreement with the "flared" limits and were entirely adequate for practical purposes.

Fig. 9, 10, 11, 12, 13, and 14 show, respectively, the relation of septal thickness, posterior left ventricular wall thickness, the internal dimension of the aortic root, right ventricular internal dimension, left ventricular internal dimension, and left atrial internal dimension to gestational age. The correlation coefficients $(r)$ ranged from 0.836 to 0.895 and these were statistically highly significant ( $t>14.968$; $p<0.001)$. Statistical data for the relation of each measurement to gestational age and the equations for the lines are given with the appropriate figure.

\section{Discussion}

Realtime imaging of the fetal cardiac anatomy has been described by several authors. ${ }^{124}$ Prenatal detection of structural cardiac abnormalities has also been reported during the middle ${ }^{56}$ and last trimesters ${ }^{4}$ of pregnancy. Others have reported that the M-mode echocardiogram can also be obtained in fetal life. ${ }^{478}$ We have described the normal appearance of the complete $M$-mode echocardiogram during fetal life and the method of obtaining it.

The M-mode echocardiogram provides complementary information to the two dimensional study. For this to be of value and to avoid errors in interpretation, it must be performed in a standard way such as that we have described. The $M$-line must be positioned by accurate two dimensional guidance and constant reference between the $M$-mode and the two dimensional image must be made as chamber orienta-

Table $95 \%$ confidence limits for measurements at 16 weeks, 39 weeks, and at mean gestational age

\begin{tabular}{|c|c|c|c|c|}
\hline & $\begin{array}{l}S E E \\
y \times 2\end{array}$ & 16 weeks & $\begin{array}{l}\text { Mean } \\
\text { gestation }\end{array}$ & 39 weeks \\
\hline Septal thickness & 0.088 & 0.0873 & 0.0867 & 0.089 \\
\hline $\begin{array}{l}\text { Posterior left ventricular } \\
\text { wall thickness }\end{array}$ & 0.066 & 0.067 & 0.066 & 0.067 \\
\hline Aortic root & 0.138 & 0.138 & 0.137 & $0.139(38)$ \\
\hline $\begin{array}{l}\text { Right ventricular internal } \\
\text { dimension }\end{array}$ & 0.306 & 0.306 & 0.304 & 0.311 \\
\hline $\begin{array}{l}\text { Left ventricular internal } \\
\text { dimension }\end{array}$ & $0 \cdot 300$ & 0.300 & 0.298 & 0.304 \\
\hline $\begin{array}{l}\text { Left atrial internal } \\
\text { dimension }\end{array}$ & 0.2995 & $\begin{array}{l}0.2969 \\
(17)\end{array}$ & 0.297 & 0.307 \\
\hline
\end{tabular}

Note: Confidence limits were not found to "flare" significantly at the extremes of gestational age. 
tion to the transducer may change during fetal movement. This is particularly so in the very active midtrimester fetus.

The M-mode echocardiogram can demonstrate that cardiac structures have a characteristic pattern of motion. It can show actual cusp separation of valve

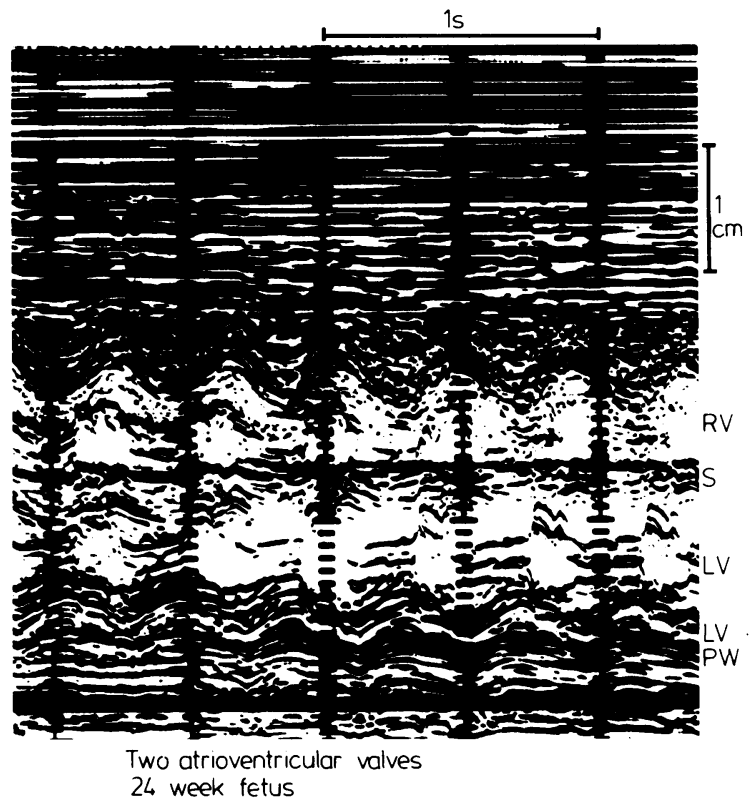

Fig. $7 A$ "sweep" between the two atrioventricular valves of each ventricle in a 24 week fetus. The tricuspid valve motion is seen opening within the right ventricle, the mitral valve within the left ventricle. leaflets where the two dimensional image may only show a moving valve which could be atretic. It can show the "box" shape in systole and sloping central closure line characteristic of both normal arterial valves. In the normal individual in postnatal life it is possible to distinguish the aortic from the pulmonary valve by their patterns of motion and by their systolic time intervals. In contrast, in the normal fetus this is not possible. The motion of both valves is indistinguishable and ejection times were similar for both aortic and pulmonary valves. Since the speed of the recordings was $50 \mathrm{~mm} / \mathrm{s}$ minor differences cannot be excluded. Recording at faster speeds, however, provided tracings that were inadequate for interpretation. Notwithstanding this reservation it appears reasonable that aortic and pulmonary systolic time intervals should be similar, since both right and left ventricles are ejecting against similar resistances.

Similarly, the two atrioventricular valves are indistinguishable by $\mathrm{M}$-mode alone and must be identified from their respective chamber. The pattern of their motion is the " $M$ " shape seen mainly in the mitral valve in postnatal life. Septal motion is always towards the left ventricular wall in systole. Other authors ${ }^{3}$ have suggested a high incidence of paradoxical septal motion in fetal life. We have never seen this, however, in a normal fetal heart. Before 20 weeks gestation septal motion is flat but after this time it has the appearance seen in Fig. 8.

The pattern of motion of the foramen ovale flap can be observed on $\mathrm{M}$-mode. On the two dimensional scan it could be seen to move throughout the cardiac cycle but when recorded in M-mode this motion could

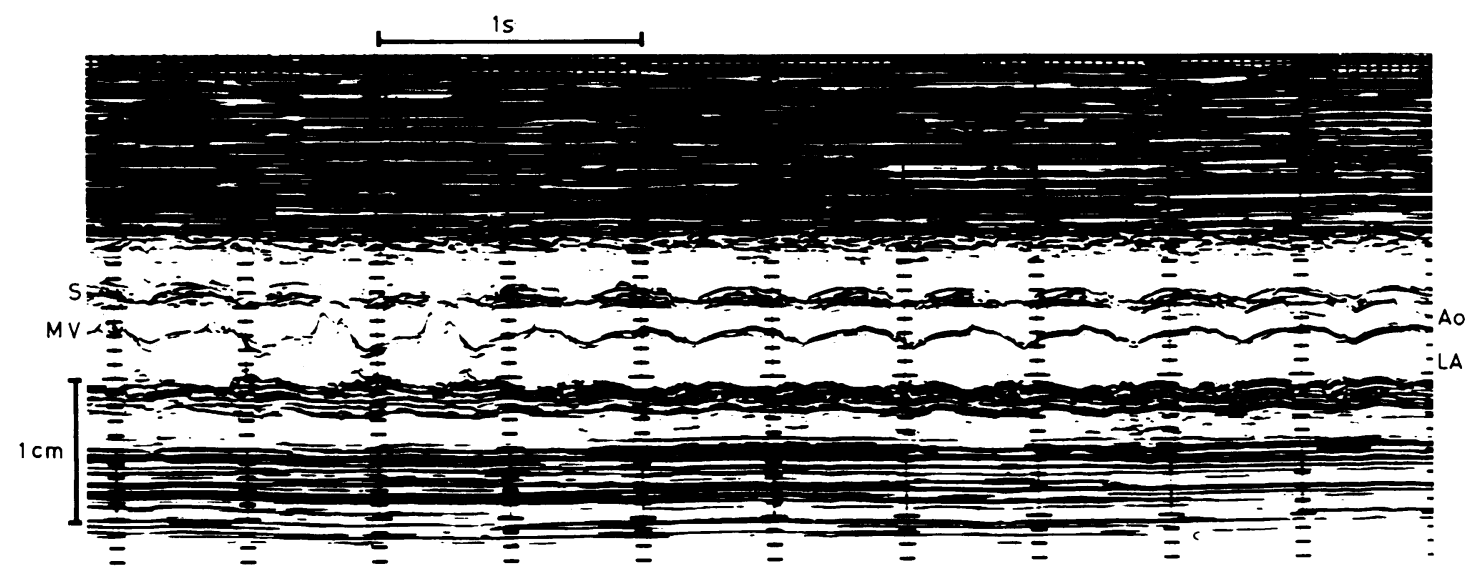

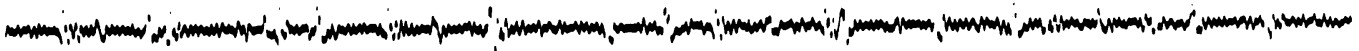

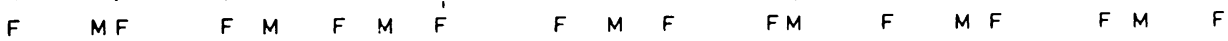

Fig. 8 This shows a mitral-aortic sweep and shows mitral-aortic and septal-aortic continutity. 
be seen to be organised. The flap approximates to the atrial septum, or closes, twice during each cardiac cycle (Fig. 4). This movement pattern is consistently recordable in every normal fetal heart. When this motion was timed with the fetal electrocardiogram and left atrial wall motion there appeared to be an abrupt closure of the foramen ovale flap during atrial systole; it then opened more gradually and then drifted closed during ventricular systole. Closure during ventricular systole may be the result of the effect

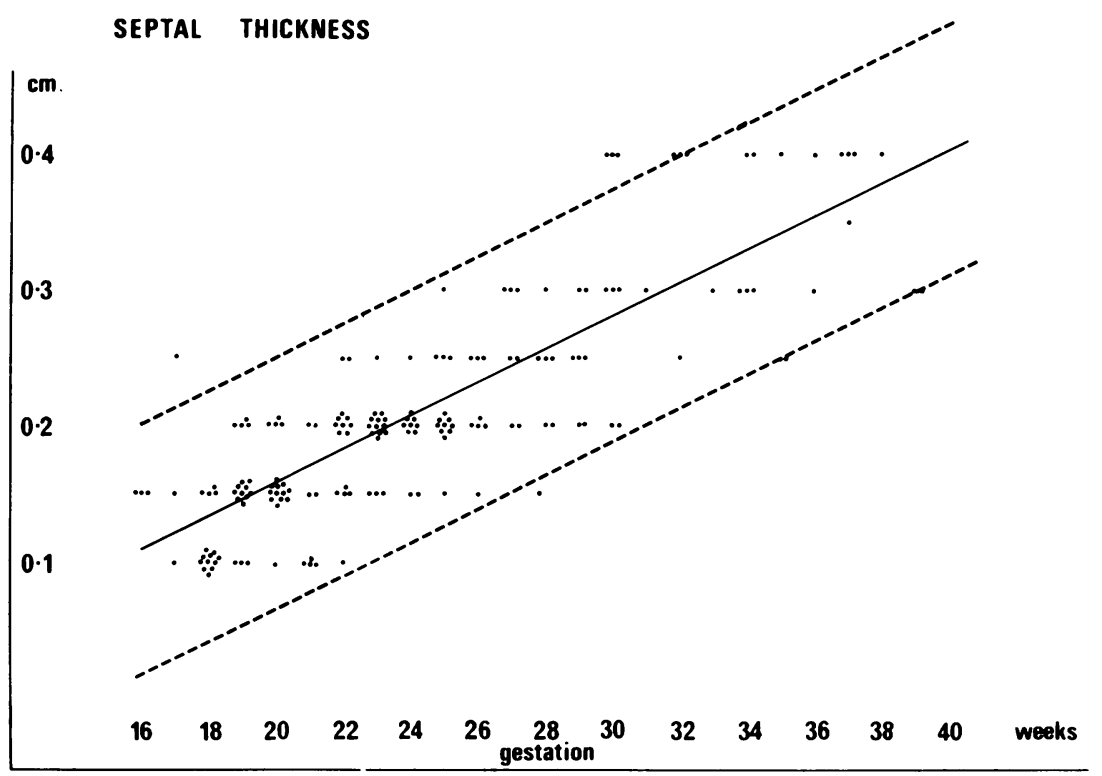

Fig. 9 Septal thickness plotted against gestational age $(n=178)$. The $95 \%$ confidence limits represent twice the standard error of the mean in each graph. $Y=0.012 \times-0.088, r=0.836, t=20.183$, $p<0.001$, SEE $y \times 2=0.088$.

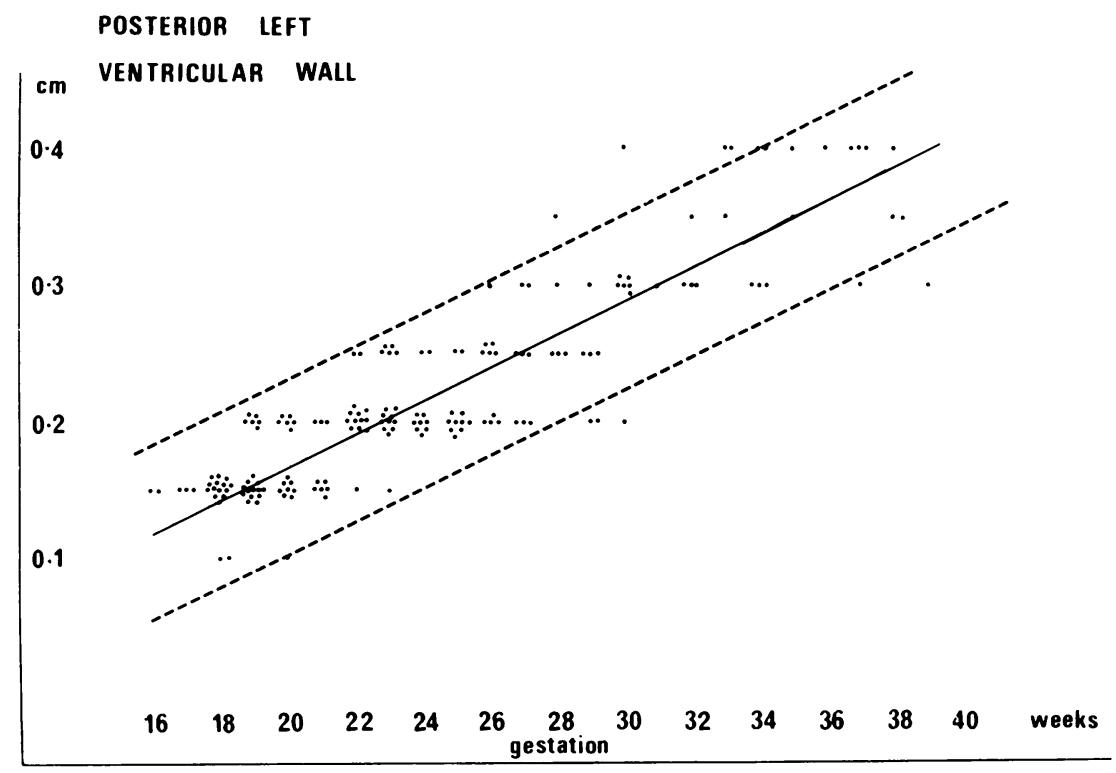

Fig. 10 Posterior left ventricular wall thickness plotted against gestational age ( $n=175$ ). $Y=0.012 \times-0.063, r=0.884, t=24.929, p<0.001, S E E y \times 2=0.66$. 
of the pulmonary venous return to the left atrium, small though it may be, meeting a closed mitral valve. Once ventricular systole ends the foramen ovale flap opens again. This suggests a biphasic pattern of flow from the right to the left atrium related in part to atrial systole but also being determined by the relative systemic and pulmonary venous returns. The factors that produce this sequence are at present speculative and merit further study.

From the growth charts it can be seen that septal and left ventricular wall thickness grow in a parallel fashion throughout pregnancy, increasing between three and fourfold during gestation. The left and right ventricular dimensions again parallel each other until

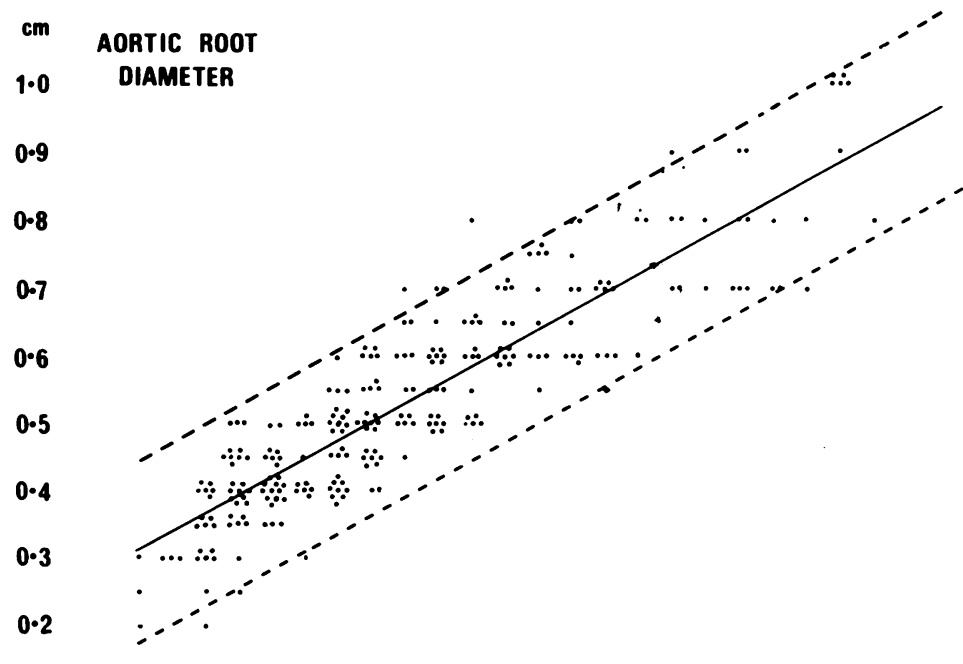

0.1

$$
\begin{array}{llllllllllllll}
16 & 18 & 20 & 22 & 24 & 26 & 28 & 30 & 32 & 34 & 36 & 38 & 40 & \text { weeks }
\end{array}
$$

Fig. 11 Aortic root diameter plotted against gestational age $(n=254) . Y=0.028 \times-0.136$, $r=0.895, t=31.925, p<0.001$, SEE $y \times 2=0.138$.

\section{RIGHT VENTRICULAR \\ INTERNAL DIMENSION}

cm

$2 \cdot 0$

1.5

$1 \cdot 0$

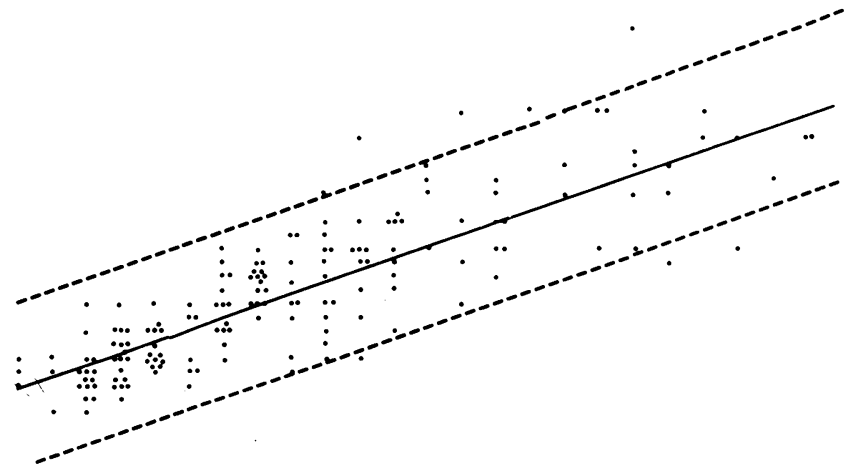

0.5

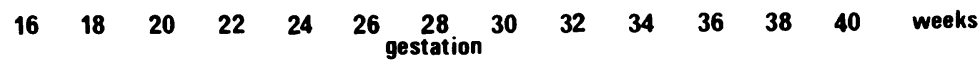

Fig. 12 Right ventricular internal dimension plotted against gestational age $(n=167)$. $Y=0.045 \times-0.228, r=0.844, t=20.183, p<0.001, S E E y \times 2=0.306$. 


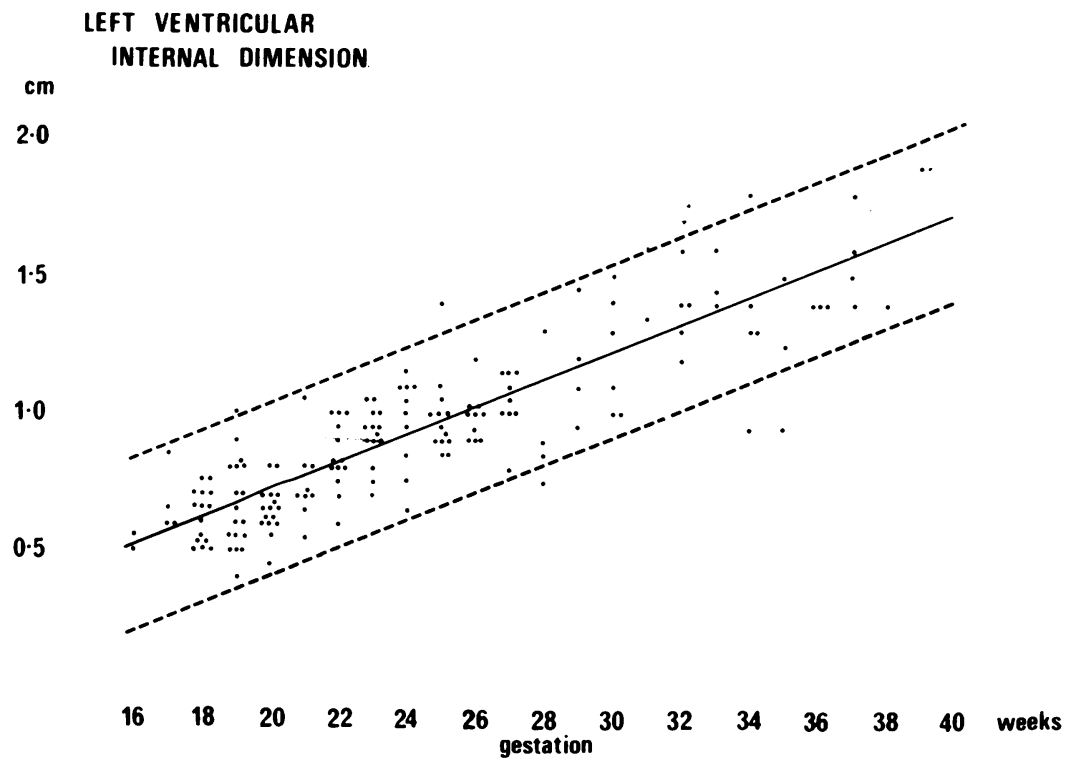

Fig. 13 Left ventricular internal dimension plotted against gestational age $(n=175)$. $Y=0.049 \times-0.262, r=0.876, t=23.944, p<0.001, S E E y \times 2=0.3$.

LEFT ATRIAL

cm

INTERNAL DIMENSION

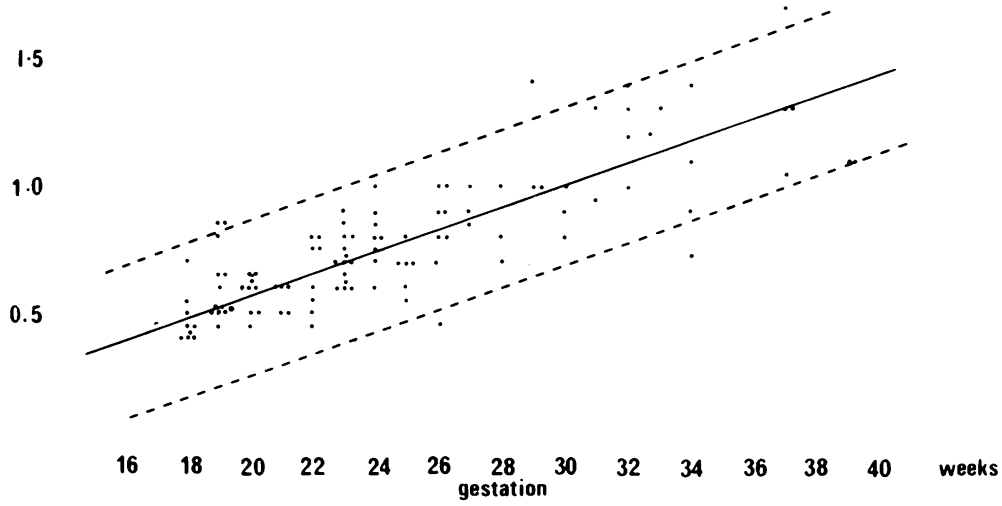

Fig. 14 Left atrial internal dimension plotted against gestational age $(n=107)$. $Y=0.040 \times-0.214, r=0.823, t=14.968, p<0.001$, SEE $y \times 2=0.296$.

term, bearing approximately a one to one relation to each other throughout gestation. Again this may represent equal pressure within the two chambers and equal volumes handled by each chamber. The mean internal diameter of each at 16 weeks gestation is about $0.5 \mathrm{~cm}$, increasing to a mean of approximately $1.5 \mathrm{~cm}$ at term. The left ventricular dimension at term compares well with published neonatal left ventricular dimensions. ${ }^{9}$ It should be noted, however, that our left ventricular internal dimension is not strictly enddiastolic as has been explained in the text but is fractionally larger as it represents the maximum internal dimension. The left atrial and aortic root dimensions also show similar growth patterns, but it is interesting that the ratio of left atrial to aortic root dimension is consistently greater than unity, being approximately 
1.5:1. Since the fetal aortic root dimensions at term are similar to those of newborn infants, this suggests that in the fetus the left atrium is enlarged compared with postnatal life. Examination of Fig. 15 perhaps explains this fact because it can be seen that the Eustachian valve is almost completely preventing the passage of inferior vena caval blood through the tricuspid valve; instead the flow is mainly directed through the foramen ovale into the left atrium. The inferior vena cava in fetal life carries all the venous flow from the lower half of the body and also the venous return from the placenta. These anatomical and functional features of the fetal heart have been well described in animal specimens, especially in lambs. Some observers of the fetal lamb suggest that left ventricular output is greater than the right ${ }^{10}$ though others find similar outputs from each ventricle. ${ }^{11}$

The relative left atrial enlargement that has been observed in these normal human fetuses suggests that left atrial flow is high. This supports the concept that left ventricular output may be greater than that of the right ventricle. This is, however, in conflict with the data indicating similar right and left ventricular dimensions and similar aortic and pulmonary valve systolic time intervals. Thus, further studies of the normal human fetus are required to elucidate the normal fetal haemodynamics.
In all the measurement data the best fit correlation between dimensions and gestational age was a straight line. Inability to detect a curvilinear relation may be because we are observing growth at its most rapid phase. It may be, however, that when more data have been accumulated in older fetuses the growth charts may need modification, but at present they give a good indication of normal dimensions and as such should be clinically useful.

Dimensional data obtained from normal fetuses should be valuable in the interpretation of abnormalities of the fetal heart. For example, if the right ventricle is larger than the left it is useful to know whether the right ventricle is dilated or the left ventricle is small for the gestational age. A single measurement out of step with the others, however, might lead one to suspect a structural cardiac abnormality. The growth charts shown here should not be used for gestational age dating as this is more accurately achieved by other means. They may, however, be useful in the more detailed study of the effects of growth retardation on the heart. In such cases all the measured variables might be expected to show a similar shift to the left.

In summary, familiarity with the appearance of the normal M-mode echocardiogram and the growth of cardiac structures in the normal fetus should help in

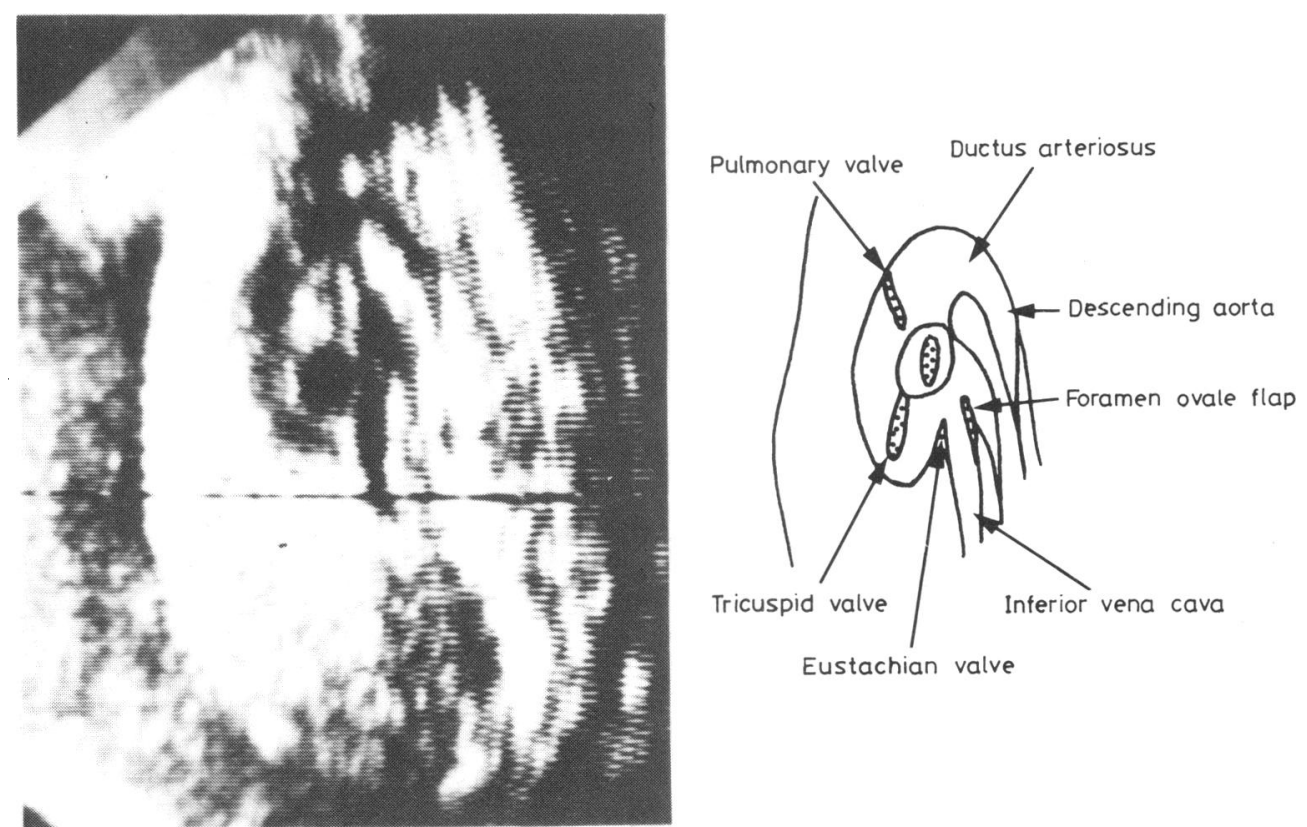

Fig. 15 The fetal heart is visualised in the ductal plane. The aortic valve is seen in the centre. The inferior vena cava can be seen entering the right atrium but the Eustachian valve and the foramen ovale flap can be seen to direct most of the flow to the left atrium. 
the elucidation of structural cardiac abnormalities in fetal life. Further study should aid understanding of cardiac function in the normal human fetus.

The author would like to thank Dr A P Roberts of the Department of Medical Microbiology, West London Hospital, for the statistical analysis.

\section{References}

1 Sahn DJ, Lange L, Allen HD, et al. Quantitative real time cross-sectional echocardiography in the developing normal human fetus and newborn. Circulation 1980; 62: 588-97.

2 Allan LD, Tynan MJ, Campbell S, Wilkinson JW, Anderson RH. Echocardiographic and anatomical correlates in the fetus. Br Heart $\mathcal{F} 1980$; 44: 444-51.

3 Campbell S, Newman GB. Grown of fetal biparietal diameter during normal pregnancy. Foumal of Obstetrics and Gynaecology of the British Commonwealth 1971; 78: 513-9.

4 Kleinman CS, Hobbins JC, Jaffe CC, Lynch DC, Talner NS. Echocardiographic studies of the human fetus: prenatal diagnosis of congenital heart disease and cardiac dysrhythmias. Pediatrics 1980; 65: 1059-67.
5 Allan LD, Tynan M, Campbell S, Anderson RH. Identification of congenital cardiac malformations by echocardiography in midtrimester fetus. $\mathrm{Br}$ Heart $\mathcal{F}$ 1981; 46: 358-62.

6 Henrion R, Aubry JP. Fetal cardiac abnormality and real-time ultrasound study: a case of Ivemark syndrome. Contrib Gynecol Obstet 1979; 6: 119-22.

7 Winsberg F. Echocardiography of the fetal and newborn heart. Invest Radiol 1972; 7: 152-8.

8 Baars AM, Merkus JMWM. Fetal echocardiography: a new approach to the study of the dynamics of the fetal heart and its component parts. Eur $\mathcal{F}$ Obstet Gynaecol Reprod Biol 1977; 7/2: 91-100.

9 Solinger R, Elbl F, Minhas K. Echocardiography in the normal neonate. Circulation 1973; 47: 108-18.

10 Dawes GS, Mott JC, Widdicome JG. The foetal circulation in the lamb. F Physiol 1954; 126: 563-87.

11 Mahon WA, Goodwin JW, Paul WM. Measurement of individual ventricular outputs in the fetal lamb by a dye dilution technique. Circ Res 1966; 19: 191-8.

Requests for reprints to Dr Lindsey D Allan, Guy's Hospital, St Thomas Street, London SE1 9RT. 